\title{
Article \\ Healthy Eating Is Associated with Sarcopenia Risk in Physically Active Older Adults
}

\author{
Konstantinos-Georgios Papaioannou ${ }^{1}$ D , Andreas Nilsson ${ }^{1, *(D)}$, Lena Maria Nilsson ${ }^{2}$ (D) and Fawzi Kadi ${ }^{1}$ (D) \\ 1 School of Health Sciences, Örebro University, 70281 Örebro, Sweden; \\ konstantinos.papaioannou@oru.se (K.-G.P.); fawzi.kadi@oru.se (F.K.) \\ 2 Epidemiology and Global Health, Umeå University, 90187 Umeå, Sweden; lena.nilsson@umu.se \\ * Correspondence: andreas.nilsson@oru.se; Tel.: +46-19-303-553
}

Citation: Papaioannou, K.-G.;

Nilsson, A.; Nilsson, L.M.; Kadi, F.

Healthy Eating Is Associated with

Sarcopenia Risk in Physically Active Older Adults. Nutrients 2021, 13, 2813. https://doi.org/10.3390/nu13082813

Academic Editors:

David Jiménez-Pavón and

Ana Carbonell-Baeza

Received: 21 June 2021

Accepted: 14 August 2021

Published: 16 August 2021

Publisher's Note: MDPI stays neutral with regard to jurisdictional claims in published maps and institutional affiliations.

Copyright: (c) 2021 by the authors. Licensee MDPI, Basel, Switzerland. This article is an open access article distributed under the terms and conditions of the Creative Commons Attribution (CC BY) license (https:// creativecommons.org/licenses/by/ $4.0 /)$.
Abstract: Healthy Diet and physical activity may play important roles in the maintenance of muscle health during aging. The aim of the present study was to explore the impact of adherence to healthy dietary patterns on sarcopenia risk in a sample of physically active older men and women, while considering adherence to guidelines on muscle strengthening activities (MSA) and protein intake. Based on a sample of 191 physically active men and women (65-70 years), dietary intake was assessed using a 90-items food-frequency-questionnaire (FFQ) and Healthy Diet Score (HDS) was calculated. Physical activity was assessed by accelerometry and self-report. A sarcopenia risk score (SRS) was derived based on three indicators of muscle health: muscle mass was assessed using bioelectrical impedance and handgrip strength and 5 times sit-to-stand (5-STS) were determined by standardized procedures. Analysis of covariance (ANCOVA) was used to examine differences in SRS and its components across sex-specific tertiles of HDS, with adjustments for covariates including total energy intake, protein intake and MSA. A significant main effect $(p<0.05)$ of HDS on SRS was observed, where those belonging to the highest HDS tertile had lower SRS compared to those in the lowest tertile. A corresponding significant effect was observed for 5-STS performance, with better performance in those with the highest HDS adherence compared to those with the lowest. The present study supports guidelines emphasizing diet quality beyond amounts of macro- and micronutrients in the prevention of age-related deterioration of muscle health. Importantly, the benefits from healthy dietary patterns are evident in older adults who already adhere to guidelines for health-enhancing physical activity.

Keywords: muscle mass; aging; physical function; handgrip strength; Healthy Diet Score (HDS); dietary patterns; physical activity; sarcopenia risk; diet quality; muscle strengthening activities

\section{Introduction}

Aging is accompanied by a progressive decline in skeletal muscle mass and function, which ultimately leads to an increased risk of developing sarcopenia [1], defined as a generalized skeletal muscle disorder characterized by an increased risk of falls, physical disability and mortality [1,2]. A clinical definition of sarcopenia based on the three components; muscle strength, muscle mass and measures of physical performance, has been issued by the European Working Group on Sarcopenia in Older People 2 (EWGSOP2) [1] In this respect, lifestyle behaviors, including diet and physical activity may play an important role in preventing the occurrence of sarcopenia [3-5]. Indeed, several reports have highlighted the putative role of proteins on muscle health [6], where adherence to a recommended daily intake of $0.8 \mathrm{~g} \cdot \mathrm{kg}^{-1}$ bodyweight (BW) has a beneficial impact on muscle mass [7], whereas a consumption greater than $1.1 \mathrm{~g} \cdot \mathrm{kg}$ per day is linked to both higher muscle mass and muscle function in older adults $[8,9]$. The exploration of the impact of overall dietary patterns, rather than single nutrients, on skeletal muscle health has the advantage of considering the complex interplay and potential synergistic effects by different foods on 
health outcomes. In this respect, a series of studies suggested that healthy dietary patterns, characterized by greater fruit, vegetable and wholegrain consumption, may infer beneficial effects on muscle strength and function [10]. However, less evidence is available in older adults and previous studies often considered isolated parameters of muscle function, which unlikely covers overall dimensions of muscle health [10]. Moreover, a recent systematic review reported inconsistencies in the relationship between dietary patterns and indicators of muscle health, which highlights the need for further studies investigating the role of dietary habits diet on muscle health in older adults [11]. An important step towards the understanding of the independent influence of diet on determinants of sarcopenia risk is to consider, in general, the potential myotrophic effects of physical activity (PA) habits and, more specifically, muscle-strengthening activities (MSA) [12,13]. Indeed, major health organizations, including WHO, highlight the beneficial role of MSA in promotion of muscle health in older adults [14]. Several biological factors may mediate the putative effects of dietary patterns on muscle health. For example, detrimental effects of a pro-inflammatory environment on muscle health have previously been documented [15]. Furthermore, there is an interplay between elevated inflammatory status and abdominal obesity [16], which in turn may accelerate the rate of muscle wasting by advancing age. Therefore, such biological factors need to be considered when elucidating links between healthy eating and indicators of muscle health in older adults.

The aim of the present study was to explore the impact of overall dietary patterns on components of the sarcopenia risk in a sample of physically active older men and women.

\section{Materials and Methods}

\subsection{Participants}

A total of 252 community-dwelling Swedish older adults (men and women) aged 65-70 years old were recruited through local advertisement during 2018. Exclusion criteria were: presence of overt disease including diabetes mellitus, coronary heart disease, musculoskeletal disorders, psychiatric disease and mobility disabilities. In addition, participants who accumulated less than $150 \mathrm{~min} /$ week of moderate-to-vigorous physical activity (MVPA) were excluded. A written informed consent was obtained from participants and all investigations were performed in accordance with the Declaration of Helsinki ethical principles. The Regional Ethics Committee of Uppsala, Sweden, approved the study (Dnr 2017/511).

\subsection{Assessment of Dietary Intake}

Dietary data were collected using a 90-items food-frequency questionnaire (FFQ) based on a validated 84- and 65-items FFQ, developed within the Västerbotten Intervention Programme (VIP) $[17,18]$. All participants received instructions face-to-face by a single investigator prior to filling out the FFQ. The FFQ has 9 fixed alternatives (never, occasionally, 1-3 times/month, 1 time/week, 2-3 times/week, 4-6 times/week, 1 time/day, 2-3 times/day, $\geq 4$ times/day) for assessing the intake frequency. The Healthy Diet Score (HDS), was derived on the basis of reported intakes of favorable (fish, fruits (except juices), vegetables (except potatoes) and whole grains) and unfavorable (red or processed meats, desserts and sweets, sugar-sweetened beverages and fried potatoes) food groups [19]. The frequency intake of each food group was ranked in sex-specific quartiles and assigned an ascending $(0,1,2,3)$ or descending $(3,2,1,0)$ value, for favorable and unfavorable food groups, respectively. The quartile ranks were summed into the Healthy Diet Score (min 0; max 24), where a higher rank indicates healthier dietary pattern. Based on HDS, participants were divided into sex-specific tertiles reflecting adherence to a healthy diet (Low, Moderate and High). In addition, total energy intake and daily protein intake were retrieved. The protein cut-point of $1.1 \mathrm{~g} \cdot \mathrm{kg}^{-1} \mathrm{BW}$ was used to determine adherence to guidelines on protein intake in older adults [20]. 


\subsection{Assessment of Anthropometry and Components of Sarcopenia Risk}

Standard anthropometrical procedures were used to assess body height and weight. Waist circumference (WC) was measured at the midpoint between the lower costal margin and the iliac crest using measuring tape. Bioelectrical impedance analysis (Tanita MC-780, Tanita Amsterdam, The Netherlands) was used to assess skeletal muscle mass index (SMI). Skeletal muscle mass (SMM) was computed using the Janssen equation [21], and expressed as a percentage of body weight (SMI, \%). Indices of muscle function were assessed as follows: handgrip strength (HG) using a Jamar hand dynamometer (Patterson Medical, Warrenville, IL, USA) and the five times sit-to-stand test (5STS), where participants were instructed to sit down in a chair, starting from a standing upright position and to repeat this sequence 5 times. Based on current operational definition issued by the EWGSOP2 [1] sarcopenia is clinically diagnosed based on an integration of the following components: low muscle strength and low muscle mass, and low physical performance as an indicator of severity. Therefore, in line with this definition and in accordance with a large body of previous research, we aggregated a standardized sex-specific score denoting sarcopenia risk according to the following: firstly, the three variables were standardized (z-scores) in men and women separately. Secondly, the three sex-specific standardized variables were averaged into one single SRS variable. Importantly, the SRS cannot classify whether individuals have sarcopenia, but rather denotes higher or lower risk of sarcopenia based on an aggregation of sarcopenia risk components as used in previous work [22].

\subsection{Assessment of Adherence to PA Guidelines}

The Actigraph GT3x (Actigraph, Pensacola, FL, USA) accelerometer was used to assess adherence to the PA guideline regarding 150 weekly min of MVPA, as previously described [23]. Briefly, participants were instructed to wear the monitor around the waist during all waking hours (except water-based activities) for a period of seven days. A minimum of four days with at least $10 \mathrm{~h}$ of wear time per day was required for inclusion. Non-wear time was defined as a minimum of $60 \mathrm{~min}$ of continuous zero accelerometer counts. Time spent in MVPA was determined based on the established accelerometer count cut-point of $>2019$ counts per minute [24]. Participants accumulating an average of $22 \mathrm{~min}$ of MVPA per day (approximating 150 min per week) were classified as meeting current guidelines about health-enhancing PA issued by the WHO [14]. Adherence to MSA was assessed using the EPAQ2 questionnaire [25] where participants reported on duration and frequency of MSA during the last 12 months. Participants were then stratified into two groups ( $\geq 2$ sessions per week or less).

\subsection{Assessment of High-Sensitivity C-Reactive Protein (hs-CRP)}

Blood samples were collected from an antecubital vein after an overnight fast. Subjects were asked not to engage in any strenuous physical activity and avoid smoking and alcohol $24 \mathrm{~h}$ before the sampling. An automated immunoturbidimetric assay (Advia 1800, Chemistry System, Siemens, Germany) using a Hs-CRP kit was employed to assess Serum CRP level.

\subsection{Statistical Analysis}

Data are presented as means $\pm \mathrm{SD}$, unless otherwise stated. Normal distribution was checked by visual inspection and Shapiro-Wilks tests and data was log-transformed when necessary, to fit a normal distribution. Sex-differences in general characteristics were assessed using independent samples T-test. Analysis of covariance (ANCOVA) was used in order to determine the effects on components of SRS across tertiles of HDS. First, analysis was adjusted by total energy intake and thereafter the adherence to guidelines for protein intake (yes/no) and to the MSA guideline (yes/no) were added as fixed factors, with waist circumference and level of CRP as continuous covariates. In addition, post-hoc tests with Bonferroni correction were performed to examine the differences between tertiles. A priori power calculation indicated the detection of small to moderate effect sizes on SRS with 
a power of $\geq 80 \%$ when based on the study sample size and an alpha level of 0.05 . All statistical analyses were performed using SPSS Statistics, version 27.0 (IBM Corp., Armonk, NY, USA).

\section{Results}

The final analyses included a total of 69 men $(67.4 \pm 1.5$ years $)$ and 122 women (67.4 \pm 1.6 years) who accumulated $150 \mathrm{~min} /$ week of MVPA and had complete data on all variables. Significant sex-specific differences were observed for all anthropometric variables and HG, whereas no such difference was observed for 5STS performance (Table 1).

Table 1. Anthropometrics and components of the sarcopenia risk score (SRS).

\begin{tabular}{ccc}
\hline & Men & Women \\
\hline Anthropometrics & & \\
Height $(\mathrm{cm})$ & $178.2 \pm 6$ & $164.6 \pm 5.8^{*}$ \\
Weight $(\mathrm{kg})$ & $79.9 \pm 11.1$ & $63.4 \pm 9.0^{*}$ \\
BMI $\left(\mathrm{kg} / \mathrm{m}^{2}\right)$ & $25.1 \pm 3.0$ & $23.4 \pm 3.2^{*}$ \\
Waist circumference $(\mathrm{cm})$ & $93.4 \pm 9.9$ & $78.9 \pm 8.5^{*}$ \\
$\quad$ Sarcopenia Risk & & \\
Skeletal Mass Index (\%) & $34.6 \pm 3.1$ & $26.9 \pm 3.4^{*}$ \\
Hand grip per body weight (g.kg-1 bodyweight) & $0.56 \pm 0.10$ & $0.45 \pm 0.08^{*}$ \\
5 Sit-to-Stand test (s) & $10.1 \pm 1.9$ & $10.2 \pm 2.3$ \\
\hline
\end{tabular}

Data are expressed as mean $\pm \mathrm{SD} .{ }^{*} p<0.05$ between sexes.

There were no sex-differences in CRP levels (men: $1.0 \pm 2.6 \mathrm{mg} / \mathrm{L}$ vs. women: $1.0 \pm 2.5 \mathrm{mg} / \mathrm{L})$. Total energy intake was significantly higher in men (2146 $\pm 612 \mathrm{kcal}) \mathrm{com}-$ pared to women $(1540 \pm 472 \mathrm{kcal})$, whereas no corresponding differences were observed in relative protein intake (men: $1.01 \pm 0.32 \mathrm{~g} \cdot \mathrm{kg}^{-1} \mathrm{BW}$ vs. women: $0.99 \pm 0.31 \mathrm{~g} \cdot \mathrm{kg}^{-1} \mathrm{BW}$ ). Median intakes of the eight HDS food items are presented stratified by tertiles of HDS adherence in Table 2.

Table 2. Intakes (g/day) of the eight components of the Healthy Diet Score (HDS) stratified by tertiles of HDS.

\begin{tabular}{cccc}
\hline & Low HDS $(\boldsymbol{n}=\mathbf{6 9})$ & Moderate HDS $(\boldsymbol{n}=\mathbf{6 1})$ & High HDS $(\boldsymbol{n}=\mathbf{6 1})$ \\
\hline Favorable food groups & & & \\
Vegetables & $81.5(36.7-148.5)$ & $125.2(75.8-200.6)$ & $176.6(113.4-247)$ \\
Fruit & $110.6(70.6-196.4)$ & $165.4(90.3-239.7)$ & $236.8(161.9-317.2)$ \\
Fish & $24.8(20.3-32.7)$ & $29.5(18.1-45.0)$ & $35.9(24-46.3)$ \\
Whole grain & $55.9(35.1-68.0)$ & $57.8(38.4-78.4)$ & $67.4(50-91.4)$ \\
Unfavorable food groups & & $19.1(1.3-89.7)$ & $12.6(1-54.3)$ \\
Sugar sweetened beverages & $34.5(12.7-107.1)$ & $48.5(29.7-62.9)$ & $36.1(13.4-54.8)$ \\
Red/processed meat & $55.1(41.8-74.7)$ & $11.1(7.4-23.0)$ & $9.7(5.0-13.1)$ \\
Desserts and sweets & $14.4(9.6-28.7)$ & $8.2(0.5-17.1)$ & $8.2(0.53-13.7)$ \\
Fried potatoes & $14.0(8.0-21.4)$ & & \\
\hline
\end{tabular}

Data are expressed as median (IQR).

To examine the effect of HDS on components of SRS sarcopenia risk, we first analyzed differences across tertiles of HDS adherence, while controlling for total energy intake. Our analysis revealed a significant main effect $(p<0.05)$ of HDS on SRS. We further adjusted the model by adherence to protein and MSA guidelines as well as waist circumference and CRP levels, which left the beneficial impact of HDS on SRS significant $(p<0.05)$. Post-hoc analyses revealed a significantly lower SRS in those with highest HDS adherence compared to those with the lowest adherence (Figure 1). 


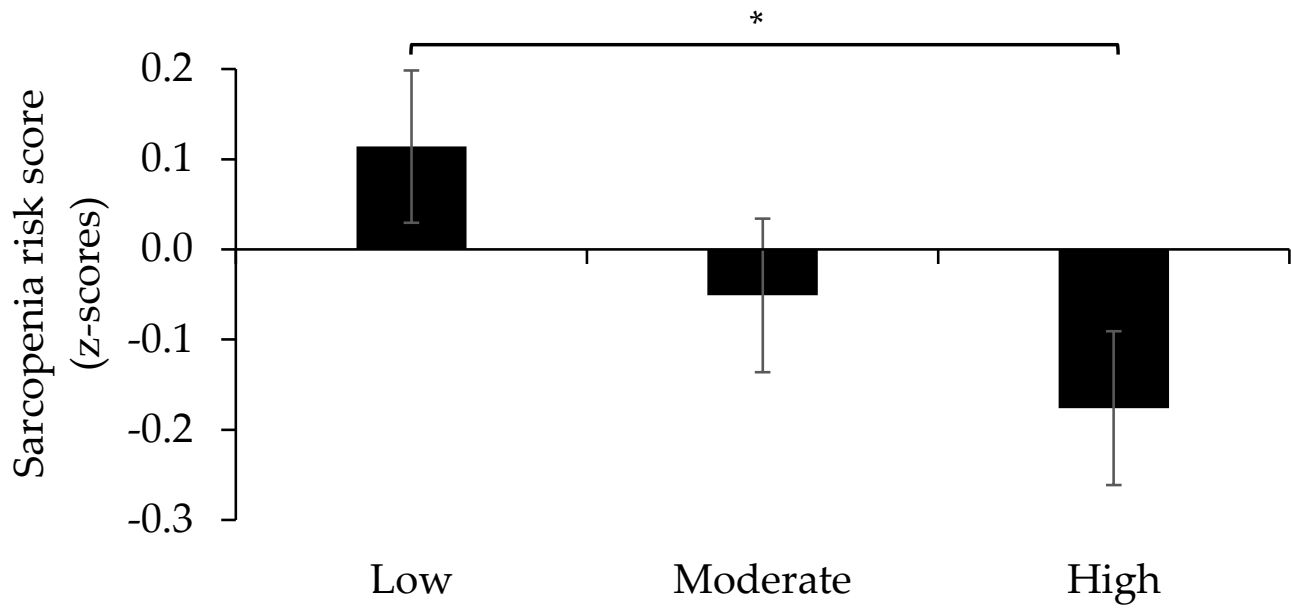

Figure 1. Sarcopenia risk score (SRS) across tertiles of the Healthy Diet Score (HDS). ${ }^{*} p<0.05$.

We further explored the impact of HDS on each single component of SRS and found significant differences in 5STS across HDS tertiles in covariate adjusted models, where those with highest HDS adherence had a better 5STS $(p<0.05)$ performance compared to those with the lowest adherence (Figure 2). Although main effects of HDS on SMI and HG were non-significant, similar trends to that observed for 5STS were indicated (Figures 3 and 4).

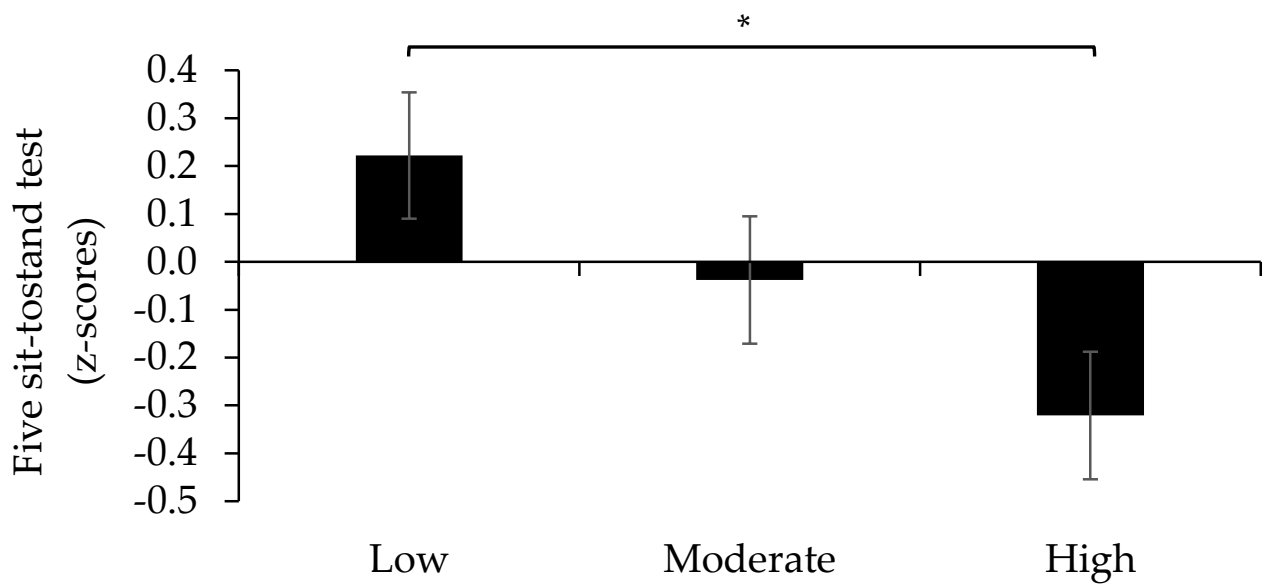

Figure 2. Five sit-to-stand test (5STS) performance across tertiles of the Healthy Diet Score (HDS). A higher score denotes lower 5-STS performance. ${ }^{*} p<0.05$.

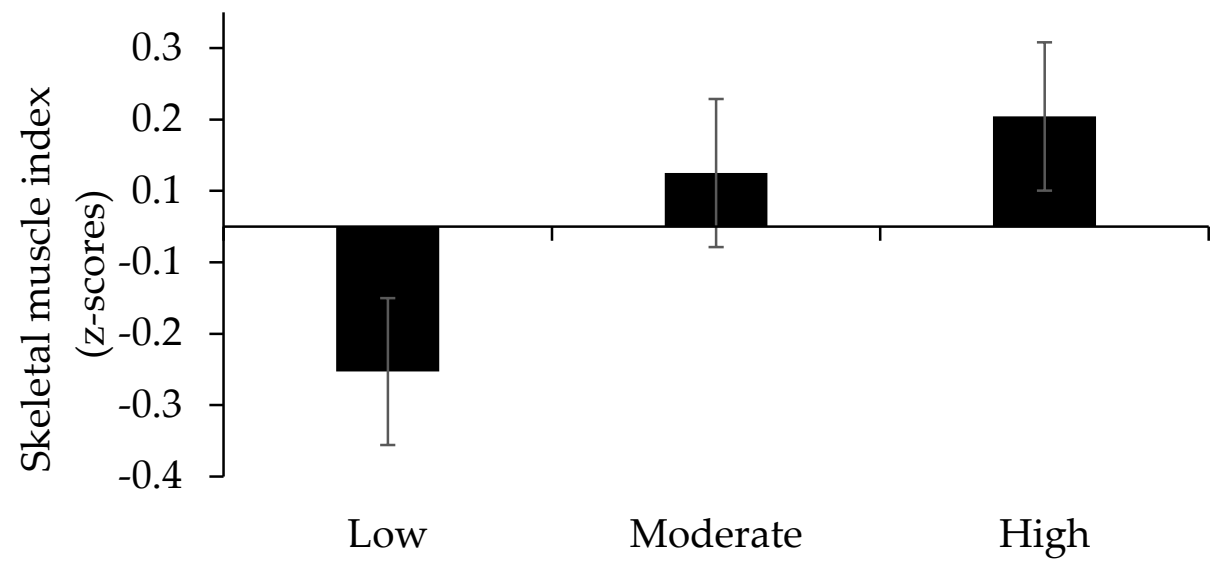

Figure 3. Skeletal muscle mass index (SMI) across tertiles of the Healthy Diet Score (HDS). A lower score denotes lower SMI. 


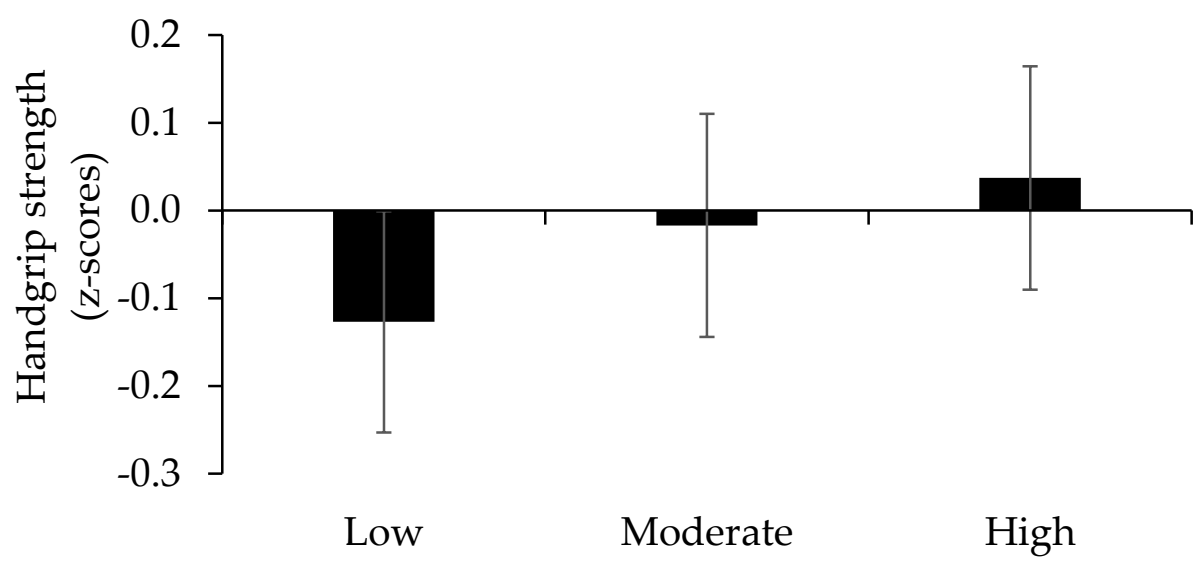

Figure 4. Handgrip strength (HG) across tertiles of the Healthy Diet Score (HDS). A lower score denotes lower HG.

\section{Discussion}

The present study highlights the beneficial link between healthy eating and sarcopenia risk in physically active older men and women. Importantly, this finding was evident even when the engagement in muscle strengthening activities and adherence to guidelines for protein intake were taken into consideration, suggesting an important role of diet quality as an independent factor able to mitigate sarcopenia risk in older adults.

Our study extends previous work on the role of overall diet quality on muscle health $[11,26-30]$ in a sample of physically older men and women, where all participants adhered to the guideline about weekly MVPA time. Given that reduced muscle activity decreases muscle mass in older adults [31] and that engagement in muscle strengthening activities lowers sarcopenia risk in older adults [13], our findings suggest that adherence to a healthy diet has beneficial effects on sarcopenia risk beyond those inferred by adherence to guidelines regarding MVPA time and engagement in muscle strengthening activities. Moreover, the observed link between HDS and SRS was independent of both total macronutrient intake and adherence to guidelines for protein intake, which suggests that dietary quality can elicit beneficial impacts on the preservation of muscle health that are separate from those driven by adequate amounts of daily protein intake. Indeed, adequate protein intake is an established core dietary element linked to muscle mass and function [22,32] and an intake of at least $1.1 \mathrm{~g} \cdot \mathrm{kg}^{-1} \mathrm{BW}$ infers beneficial effects on muscle mass and muscle function in older adults [9]. Therefore, in addition to the importance of protein intake, particular attention should be paid to the overall diet quality when conceptualizing nutritional approaches aiming to mitigate sarcopenia risk in older adults.

Several presumed mechanisms have been put forward to account for the beneficial effects of healthy diets on skeletal muscle. It is currently hypothesized that the overall health benefits of healthy eating are partly due to the modulation of the systemic inflammatory environment [33]. In this respect, serum level of CRP is an established marker of systemic inflammation and is recognized as an important factor underlying several age-related deleterious changes including muscle wasting [15]. However, a notable finding in our study was that variations in CRP levels did not affect the proposed effects of adherence to a healthy diet on sarcopenia risk. Thus, our findings indicate that the observed links between dietary patterns and SRS are not influenced by variations in CRP levels. However, our sample of physically active older men and women are likely to benefit from the MVPArelated attenuation of systemic inflammation as indicated in a previous work [34], which may mask the involvement of the inflammatory environment on links between dietary patterns and muscle health. Further investigations, preferably encompassing a wider set of inflammatory biomarkers, are warranted in order to elucidate the complex interplay between the inflammatory environment, muscle health and lifestyle behaviors. It is also hypothesized that healthy diets rich in fruit and vegetable may protect against metabolic 
acidosis and reduce proteolysis and amino acid catabolism [35], thus mitigating sarcopenia risk [36]. Additionally, an unfavorable dietary pattern, including foods rich in saturated fats may be detrimental for maintenance of muscle health [37], whereas diets rich in fiber may mitigate sarcopenia risk [38]. Altogether, the impact of dietary patterns on muscle function of older adults are unlikely to be explained by the action of single nutrients or single biological pathways. Further research is warranted in order to understand how healthy dietary patterns influence age-related decline in muscle health.

Our findings also indicate that impacts of HDS on muscle health were identified when assessing an indicator of lower muscle strength and performance but not skeletal muscle mass. Importantly, it may be hypothesized that any myotrophic effects of healthy eating are less likely to be detected in our sample of older adults, who already adhere to the general guidelines of time in MVPA, compared to less active groups of older adults. However, although not significant, trends indicating beneficial impacts of adherence to HDS on SMI and HG could, nevertheless, be observed, which warrants further investigations, preferably based on larger samples.

The strength of the present study is the adjustment of findings by important variables with the potential to influence on components of sarcopenia risk, which contributes to the robustness of the findings. The VIP FFQ has been validated both in its long 84 food item version [17], and in the short 64 food-item version which includes merged categories from the 84 food item version, at least with regards to the intake of folate and vitamin $B$ [18]. It is generally agreed that the validity of FFQs increases by the number of food items included [39]. Thus, there is no reason to believe that the validity of our FFQ would be negatively affected by adding 6 extra food items. Indeed, the exploration of the impacts of HDS has been performed while considering both the influences of adherence to the guidelines for protein intake and engagement in muscle strengthening activities, both well-established factors contributing to muscle health. However, the study is not without limitations. For example, directions of associations cannot be determined given the crosssectional design. Further, in order to address the question of whether healthy diet has an impact on SRS beyond those inferred by health-enhancing time spent in MVPA, the selected sample of older adults is unlikely to be representative of a larger sample of older adults where there is a greater variation of ethnicity, socio-demographic backgrounds, and health status. Furthermore, although several important covariates have been considered, residual confounding may still exist. Finally, despite the highest accuracy of computed tomography and magnetic resonance imaging, skeletal muscle mass was assessment by the use of bioelectrical impedance (BIA) analysis, which is more feasible for larger-scale studies. Noteworthy, in standardized conditions and using previously cross-validated equations, the BIA method is currently considered as a valid tool for measurement of functioning muscle mass in clinical settings as also acknowledged by the European Working Group on Sarcopenia in Older People 2 (EWGSOP2) [1].

\section{Conclusions}

In conclusion, the present study highlights the impact of healthy eating on components of sarcopenia risk in physically active community-dwelling older adults. This finding supports guidelines emphasizing diet quality beyond amounts of macro- and micronutrients in the prevention of age-related deterioration of muscle health. Our study also supports the promotion of healthy eating in older adults who already adhere to guidelines for health-enhancing physical activity.

Author Contributions: Conceptualization, K.-G.P., A.N. and F.K.; methodology, K.-G.P., A.N. and F.K.; validation, K.-G.P.; formal analysis, K.-G.P., A.N., L.M.N. and F.K.; investigation, K.-G.P., A.N. and F.K.; writing-original draft preparation, K.-G.P.; writing—review and editing, K.-G.P., A.N., L.M.N. and F.K.; supervision, A.N., F.K. and L.M.N.; project administration, A.N. and F.K.; funding acquisition, F.K. All authors have read and agreed to the published version of the manuscript. 
Funding: This research was funded by the EU HORIZON 2020 Research and Innovation Programme (European Joint Programming Initiative "A healthy diet for a healthy life" "JPI HDHL" and the ERA-NET co-fund HDHL-INTIMIC) GA no. 727565.

Institutional Review Board Statement: The study was conducted according to the guidelines of the Declaration of Helsinki, and approved by the Regional Ethics Committee of Uppsala, Sweden (Dnr 2017/511).

Informed Consent Statement: Informed consent was obtained from all subjects involved in the study.

Data Availability Statement: Data supporting reported results are available upon reasonable request and in accordance to the ethical principles.

Acknowledgments: The authors would like to express their sincere appreciation to Adrian Meehan for his insightful comments and Oscar Bergens for his valuable assistance in participant recruitment and collection of data, together with other colleagues involved in the research project. We thank the European Commission through the Marie Skłodowska-Curie Actions, Cofunding of Regional, National and International Programs (MSCA COFUND) for supporting KGP.

Conflicts of Interest: The authors declare no conflict of interest. The funders had no role in the design of the study; in the collection, analyses, or interpretation of data; in the writing of the manuscript, or in the decision to publish the results.

\section{References}

1. Cruz-Jentoft, A.J.; Bahat, G.; Bauer, J.; Boirie, Y.; Bruyère, O.; Cederholm, T.; Cooper, C.; Landi, F.; Rolland, Y.; Sayer, A.A.; et al. Sarcopenia: Revised European consensus on definition and diagnosis. Age Ageing 2019, 48, 16-31. [CrossRef]

2. Bahat, G.; Tufan, A.; Tufan, F.; Kilic, C.; Akpinar, T.S.; Kose, M.; Erten, N.; Karan, M.A.; Cruz-Jentoft, A.J. Cut-off points to identify Sarcopenia Accord. to European Working Group on Sarcopenia in Older People (EWGSOP) definition. Clin. Nutr. 2016, 35, 1557-1563. [CrossRef] [PubMed]

3. Makanae, Y.; Fujita, S. Role of exercise and nutrition in the prevention of sarcopenia. J. Nutr. Sci. Vitaminol. 2015, 61, S125-S127. [CrossRef]

4. Anton, S.; Hida, A.; Mankowski, R.; Layne, A.; Solberg, L.; Mainous, A.; Buford, T. Nutrition and Exercise in Sarcopenia. Curr. Protein Pept. Sci. 2017, 17. [CrossRef] [PubMed]

5. Marcos-Pardo, P.J.; González-Gálvez, N.; López-Vivancos, A.; Espeso-García, A.; Martínez-Aranda, L.M.; Gea-García, G.M.; Orquín-Castrillón, F.J.; Carbonell-Baeza, A.; Jiménez-García, J.D.; Velázquez-Díaz, D.; et al. Sarcopenia, diet, physical activity and obesity in european middle-aged and older adults: The lifeage study. Nutrients 2021, 13, 8. [CrossRef]

6. Kiesswetter, E.; Sieber, C.C.; Volkert, D. Protein intake in older people: Why, how much and how? Zeitschrift Gerontologie Geriatrie 2020, 53, 285-289. [CrossRef]

7. Beasley, J.M.; Deierlein, A.L.; Morland, K.B.; Granieri, E.C.; Spark, A. Is meeting the recommended dietary allowance (RDA) for protein related to body composition among older adults?: Results from the Cardiovascular Health of Seniors and Built Environment Study. J. Nutr. Health Aging 2016, 20, 790-796. [CrossRef] [PubMed]

8. Baum, J.; Wolfe, R. The Link between Dietary Protein Intake, Skeletal Muscle Function and Health in Older Adults. Healthcare 2015, 3, 529. [CrossRef] [PubMed]

9. Nilsson, A.; Rojas, D.M.; Kadi, F. Impact of meeting different guidelines for protein intake on muscle mass and physical function in physically active older women. Nutrients 2018, 10, 1156. [CrossRef]

10. Robinson, S.M.; Reginster, J.Y.; Rizzoli, R.; Shaw, S.C.; Kanis, J.A.; Bautmans, I.; Bischoff-Ferrari, H.; Bruyère, O.; Cesari, M.; Dawson-Hughes, B.; et al. Does nutrition play a role in the prevention and management of sarcopenia? Clin. Nutr. 2018, 37, 1121-1132. [CrossRef] [PubMed]

11. Bloom, I.; Shand, C.; Cooper, C.; Robinson, S.; Baird, J. Diet quality and sarcopenia in older adults: A systematic review. Nutrients 2018, 10, 308. [CrossRef]

12. Schoenfeld, B.J.; Ogborn, D.; Krieger, J.W. Effects of Resistance Training Frequency on Measures of Muscle Hypertrophy: A Systematic Review and Meta-Analysis. Sports Med. 2016, 46, 1689-1697. [CrossRef] [PubMed]

13. Veen, J.; Montiel-Rojas, D.; Nilsson, A.; Kadi, F. Engagement in muscle-strengthening activities lowers sarcopenia risk in older adults already adhering to the aerobic physical activity guidelines. Int. J. Environ. Res. Public Health 2021, 18, 989. [CrossRef]

14. World Health Organization. WHO Guidelines on Physical Activity and Sedentary Behaviour; World Health Organization: Geneva, Switzerland, 2020.

15. Wåhlin-Larsson, B.; Wilkinson, D.J.; Strandberg, E.; Hosford-Donovan, A.; Atherton, P.J.; Kadi, F. Mechanistic Links Underlying the Impact of C-Reactive Protein on Muscle Mass in Elderly. Cell. Physiol. Biochem. 2017, 44, 267-278. [CrossRef]

16. Bergens, O.; Nilsson, A.; Kadi, F. Cardiorespiratory Fitness Does Not Offset Adiposity-Related Systemic Inflammation in Physically Active Older Women. J. Clin. Endocrinol. Metab. 2019, 104, 4119-4126. [CrossRef] 
17. Johansson, I.; Hallmans, G.; Wikman, Å.; Biessy, C.; Riboli, E.; Kaaks, R. Validation and calibration of food-frequency questionnaire measurements in the Northern Sweden Health and Disease cohort. Public Health Nutr. 2002, 5, 487-496. [CrossRef] [PubMed]

18. Johansson, I.; Van Guelpen, B.; Hultdin, J.; Johansson, M.; Hallmans, G.; Stattin, P. Validity of food frequency questionnaire estimated intakes of folate and other B vitamins in a region without folic acid fortification. Eur. J. Clin. Nutr. 2010, 64, 905-913. [CrossRef] [PubMed]

19. Winkvist, A.; Klingberg, S.; Nilsson, L.M.; Wennberg, M.; Renström, F.; Hallmans, G.; Boman, K.; Johansson, I. Longitudinal 10-year changes in dietary intake and associations with cardio-metabolic risk factors in the Northern Sweden Health and Disease Study. Nutr. J. 2017, 16, 1-12. [CrossRef]

20. Bauer, J.; Biolo, G.; Cederholm, T.; Cesari, M.; Cruz-Jentoft, A.J.; Morley, J.E.; Phillips, S.; Sieber, C.; Stehle, P.; Teta, D.; et al. Evidence-based recommendations for optimal dietary protein intake in older people: A position paper from the prot-age study group. J. Am. Med. Dir. Assoc. 2013, 14, 542-559. [CrossRef] [PubMed]

21. Janssen, I.; Heymsfield, S.B.; Baumgartner, R.N.; Ross, R. Estimation of skeletal muscle mass by bioelectrical impedance analysis. J. Appl. Physiol. 2000, 89, 465-471. [CrossRef]

22. Montiel-Rojas, D.; Nilsson, A.; Santoro, A.; Bazzocchi, A.; de Groot, L.C.P.G.M.; Feskens, E.J.M.; Berendsen, A.A.M.; Madej, D.; Kaluza, J.; Pietruszka, B.; et al. Fighting sarcopenia in ageing european adults: The importance of the amount and source of dietary proteins. Nutrients 2020, 12, 3601. [CrossRef] [PubMed]

23. Nilsson, A.; Wåhlin-Larsson, B.; Kadi, F. Physical activity and not sedentary time per se influences on clustered metabolic risk in elderly community-dwelling women. PLoS ONE 2017, 12, e0175496. [CrossRef] [PubMed]

24. Troiano, R.P.; Berrigan, D.; Dodd, K.W.; Mâsse, L.C.; Tilert, T.; Mcdowell, M. Physical activity in the United States measured by accelerometer. Med. Sci. Sports Exerc. 2008, 40, 181-188. [CrossRef]

25. Wareham, N.J.; Jakes, R.W.; Rennie, K.L.; Mitchell, J.; Hennings, S.; Day, N.E. Validity and repeatability of the EPIC-Norfolk physical activity questionnaire. Int. J. Epidemiol. 2002, 31, 168-174. [CrossRef]

26. Na, W.; Kim, J.; Chung, B.H.; Jang, D.J.; Sohn, C. Relationship between diet quality and sarcopenia in elderly Koreans: 2008-2011 Korea national health and nutrition examination survey. Nutr. Res. Pract. 2020, 14, 352-364. [CrossRef]

27. Perälä, M.M.; Von Bonsdorff, M.B.; Männistö, S.; Salonen, M.K.; Simonen, M.; Kanerva, N.; Rantanen, T.; Pohjolainen, P.; Eriksson, J.G. The healthy Nordic diet predicts muscle strength 10 years later in old women, but not old men. Age Ageing 2017, 46, 588-594. [CrossRef] [PubMed]

28. Hashemi, R.; Motlagh, A.D.; Heshmat, R.; Esmaillzadeh, A.; Payab, M.; Yousefinia, M.; Siassi, F.; Pasalar, P.; Baygi, F. Diet and its relationship to sarcopenia in community dwelling iranian elderly: A cross sectional study. Nutrition 2015, 31, 97-104. [CrossRef] [PubMed]

29. Chan, R.; Leung, J.; Woo, J. A Prospective Cohort Study to Examine the Association Between Dietary Patterns and Sarcopenia in Chinese Community-Dwelling Older People in Hong Kong. J. Am. Med. Dir. Assoc. 2016, 17, 336-342. [CrossRef]

30. Granic, A.; Mendonça, N.; Sayer, A.A.; Hill, T.R.; Davies, K.; Siervo, M.; Mathers, J.C.; Jagger, C. Effects of dietary patterns and low protein intake on sarcopenia risk in the very old: The Newcastle 85+ study. Clin. Nutr. 2020, 39, 166-173. [CrossRef]

31. Breen, L.; Stokes, K.A.; Churchward-Venne, T.A.; Moore, D.R.; Baker, S.K.; Smith, K.; Atherton, P.J.; Phillips, S.M. Two weeks of reduced activity decreases leg lean mass and induces "anabolic resistance" of myofibrillar protein synthesis in healthy elderly. $J$. Clin. Endocrinol. Metab. 2013, 98, 2604-2612. [CrossRef]

32. Coelho-Júnior, H.J.; Milano-Teixeira, L.; Rodrigues, B.; Bacurau, R.; Marzetti, E.; Uchida, M. Relative protein intake and physical function in older adults: A systematic review and meta-analysis of observational studies. Nutrients 2018, 10, 1330. [CrossRef] [PubMed]

33. Galland, L. Diet and inflammation. Nutr. Clin. Pract. 2010, 25, 634-640. [CrossRef]

34. Nilsson, A.; Bergens, O.; Kadi, F. Physical Activity Alters Inflammation in Older Adults by Different Intensity Levels. Med. Sci. Sports Exerc. 2018, 50, 1502-1507. [CrossRef]

35. Welch, A.A. Nutritional influences on age-related skeletal muscle loss. Proc. Nutr. Soc. 2014, 73, 16-33. [CrossRef] [PubMed]

36. Kim, J.; Lee, Y.; Kye, S.; Chung, Y.S.; Kim, K.M. Association of vegetables and fruits consumption with sarcopenia in older adults: The fourth Korea national health and nutrition examination survey. Age Ageing 2015, 44, 96-102. [CrossRef]

37. Montiel-Rojas, D.; Santoro, A.; Nilsson, A.; Franceschi, C.; Capri, M.; Bazzocchi, A.; Battista, G.; de Groot, L.C.P.G.M.; Feskens, E.J.M.; Berendsen, A.A.M.; et al. Beneficial role of replacing dietary saturated fatty acids with polyunsaturated fatty acids in the prevention of sarcopenia: Findings from the nu- age cohort. Nutrients 2020, 12, 3079. [CrossRef]

38. Montiel-Rojas, D.; Nilsson, A.; Santoro, A.; Franceschi, C.; Bazzocchi, A.; Battista, G.; de Groot, L.C.P.G.M.; Feskens, E.J.M.; Berendsen, A.; Pietruszka, B.; et al. Dietary fibre may mitigate sarcopenia risk: Findings from the NU-AGE cohort of older european adults. Nutrients 2020, 12, 1075. [CrossRef]

39. Molag, M.L.; De Vries, J.H.M.; Ocké, M.C.; Dagnelie, P.C.; Van Den Brandt, P.A.; Jansen, M.C.J.F.; Van Staveren, W.A.; Van't Veer, P. Design characteristics of food frequency questionnaires in relation to their validity. Am. J. Epidemiol. 2007, 166, 1468-1478. [CrossRef] 